\title{
Evaluation of a Clinical Decision Support System in the Domain of Chronic Wound Management
}

\author{
Stefan VOGEL ${ }^{\mathrm{a}, 1}$, Jendrik RICHTER ${ }^{\mathrm{a}}$, Stefanie WACHE ${ }^{\mathrm{a}}$, Kerstin PISCHEK-KOCH ${ }^{\mathrm{a}}$, \\ Simone AUCHTER ${ }^{\mathrm{b}}$, Sebastian ZEBBITIES ${ }^{\mathrm{b}},{\text { Karen } \text { GÜTTLER }^{\mathrm{b}} \text {, Ursula HÜBNER }}^{\mathrm{c}}$, \\ Mareike PRYZSUCHA ${ }^{\mathrm{c}}$, Jens HÜSERS ${ }^{\mathrm{c}}$ and Björn SELLEMANN ${ }^{\mathrm{d}}$ \\ ${ }^{a}$ Department of Medical Informatics, Univ. Medical Center Göttingen, Germany \\ b Atacama blooms GmbH \& Co. KG, Germany \\ ${ }^{\mathrm{c}}$ Health Informatics Research Group, University AS Osnabrück, Germany \\ ${ }^{\mathrm{d}}$ Münster School of Health, Univ. of Appl. Sciences Münster, Germany
}

\begin{abstract}
The PosiThera project focuses on the management of chronic wounds, which is multi-professional and multi-disciplinary. For this context, a software prototype was developed in the project, which is intended to support medical and nursing staff with the assistance of artificial intelligence. In accordance with the user-centred design, national workshops were held at the beginning of the project with the involvement of domain experts in wound care in order to identify requirements and use cases of IT systems in wound care, with a focus on AI. In this study, the focus was on involving nursing and nursing science staff in testing the software prototype to gain insights into its functionality and usability. The overarching goal of the iterative testing and adaptation process is to further develop the prototype in a way that is close to care.
\end{abstract}

Keywords. Clinical Decision Support System, Chronic Wound, Artificial Intelligence, User-centered Design

\section{Introduction}

Clinical decision support systems (CDSS) have been developed for almost 6 decades [1, $2]$, but only few systems reach routine use $[3,4]$. A significant barrier to moving from research to routine use seems to be user acceptance of CDSS in routine use [5].

Acceptance can arise if users are involved as early as possible in the development process of a software. User-centered design is based on this concept $[6,7]$.

In the BMBF-funded PosiThera project, a CDSS was developed as a prototype in the context of chronic wound care. In the conception process, potential users, professional associations and industry were involved in national expert workshops to work out how a CDSS with artificial intelligence can assist the treatment process of a chronic wound on the IT side [8]. Different use cases were formulated from these workshops. The use cases "Diagnostic process" and "Therapy support" are addressed in

\footnotetext{
${ }^{1}$ Corresponding University Medical Center Göttingen, Department of Medical Informatics, D-37075 Göttingen, Germany; E-mail: stefan.vogel@med.uni-goettingen.de.
} 
this study in order to test the software prototype with the support of testers from the care sector and to further develop it iteratively.

\section{Methods}

Conceptually, a mixed methods design was chosen for the evaluation, with normative and descriptive parts, which is based on the black box method [9]. This method has the advantage that users can concentrate on the content and handling without having to know how to read source code.

The focus of the test is on the two use cases mentioned above, diagnostic process and therapy support, which were identified together with experts in national workshops in 2017 with regard to the meaningful implementation of AI [8]. For the evaluation of the prototype, 12 example cases were developed, which were divided into different levels of complexity. The levels of complexity were divided into simple cases, which allow an unambiguous diagnosis based on the combination of symptoms, and complex cases, which contain an ambiguous combination of symptoms and would therefore make diagnosis and therapy more difficult. Along the 12 example cases, the testers had the task to get an overview of the respective patients with the assistance of the software (wound status, monitoring of the wound, patient reported outcomes, monitoring quality of life) as well as to check the documented diagnosis of the example case with the assistance of the diagnosis support of the software.

For the use case diagnostic process, the testers evaluated whether the knowledge base of the prototype draws technically correct conclusions and whether these are displayed graphically correctly.

For the therapy support use case, the testers evaluated whether the prototype provides a manageable and practicable overview of the wound and the patient. In this context, the quality and usability of the information preparation played a major role. Furthermore, it was tested whether the monitoring of the wound available in the prototype allows conclusions to be drawn about the wound treatment. In addition, the module for recording quality of life was also included in the analysis with the question of whether the presentation can support wound treatment.

The three evaluation cycles took place in the period from February to June 2020 under laboratory conditions (Skills Lab MSH) with 5 students from the MSH Department of Health. Four of the five students have three years of nursing training and practical nursing experience and one student has paramedic training and also practical nursing experience. The process of testing and adaptation was iterative. A total of 35 tests with additional "freestyle scenarios" were carried out in 3 adaptation cycles. Freestyle scenarios were example cases that were not covered by the prepared 12 example cases and were created by the testers themselves. The assignment of the scenarios to the individual testers was done by randomised assignment. The basic requirement for the test was an internet-capable end device, which was either used privately by the testers or alternatively was available in the MSH Skills Lab. The individual test results were documented in a protocol for each participant for each test run.

For the joint exchange, 5 guiding questions were formulated, which were to be answered by each tester after the test:

1. What information about the wound would you like to be able to view in the long-term? 
2. Are there any filter functions you would like to have for monitoring? (For example, threshold values, maximum values, minimum values, trend lines, etc.).

3. In your opinion, is the sunburst diagram (see Figure 1) a suitable form to support you in diagnostics? If not, what would you like to see?

4. Is the information from the patient overview sufficient for you or would you like more/different information? (For example, symbols for deterioration or improvement of the wound status or indication of newly entered information).

5. Are there any features that you miss in the alpha demonstrator PosiThera in order to have a "rounded" support in the care of chronic wounds? If so, which ones?

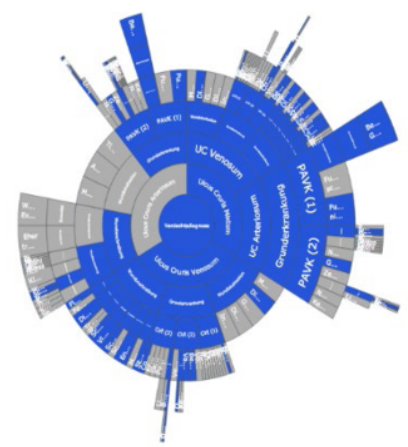

Figure 1. Snapshot of the Sunburst Diagram.

Figure 1 illustrates the sunburst diagram for the diagostic process use case. Thus, the most probable diagnoses are shown in the middle of the sunburst diagram. Documented symptoms are fanned outwards along their affiliation to the respective diagnoses and colour-coded. Thus, the blue coloured coding is the signal that specific information has been recognised and documented in the course of treatment. The greycoloured coding, on the other hand, describes that specific information has not yet been recorded. In this way, the user not only receives the recommendation of the inference as to which diagnosis is likely, but also which information may still need to be obtained in order to proceed with the treatment in a goal-oriented manner.

\section{Results}

The evaluation took place in the period January to June 2020, divided into three evaluation cycles (Cycle 1: 27.01. to 17.02.2020; Cycle 2: 02.0.3 to 26.03.2020; Cycle 3: 04.05.to 30.06.2020). In total, 35 defined fictitious patient records were evaluated by the 5 testers using the system. All testers used a PC for the evaluation. 3 testers used the Windows operating system with the Firefox browser. 2 testers performed the tests on a Mac computer using the MAC OS system and the Safari browser. 3 testers used different browsers within the 3 test cycles. Among others, the Opera browser was used for a test run as well as the Firefox browser by a Mac user. Within the three evaluation cycles, of the 35 patient records evaluated, 26 cases could be calculated correctly (74.3\%) by the CDSS and were in line with the testers' expectations. The graphical representation in the Sun burst diagram, which was used to illustrate the decision tree [10], was correct and helped the testers to get a quick overview on the symptom combination with reference 
to the suspected diagnosis. In 9 cases, no tentative diagnosis could be calculated by the system. The reason was insufficient data. In addition to the defined 35 test patient records, the testers evaluated the CDSS using so-called "freestyle scenarios". Using this method and a total of 14 freestyle scenarios, the testers mainly identified the usability of the system. For example, they were able to identify display problems with the sunburst diagram or faulty functions such as non-functioning buttons. If possible, these were corrected or fixed between the evaluation cycles.

The evaluation of the five guiding questions revealed the following picture.

Guiding question 1: One criterion mentioned by all testers was the lack of information on wound depth. In addition, the testers would have liked to receive information on the current wound healing phase (exudation, profiling, regeneration \& maturation). Furthermore, two testers mentioned the wish that the salutogenic influencing factors should also be shown in the view in addition to the pathogenic ones.

Key question 2: All 5 testers expressed the wish that the descriptive information of a wound, such as wound size and depth, amount of exudate, etc., should be displayed using a trend line or a traffic light system. This would give the observer a simpler and faster overview of the course of the wound.

Guiding question 3: The sunburst diagram was considered a suitable format by all testers. However, only at second glance, as it requires a certain amount of practice from the user. Therefore, for the correct application, training of the users regarding the sunburst diagram is required or training materials, such as videos "How do I interpret the sunburst diagram?" must be available in the system.

Guiding question 4: The information presented was rated as very comprehensive by the testers. 2 testers would like to see the infection status - if an infection is present - as additional information.

Guiding question 5: The testers wanted the already mentioned function of the trend line for a "round" support in the care, in the presentation of the descriptive information about the wound. With regard to the documentation of wound depth, one tester explicitly mentioned the "clock model" [11] for describing wound depth. Furthermore, the wish was mentioned that the information on wound care, here in particular the care materials such as wound dressings, should be included in addition to the wound healing phase. For easier documentation in the system, the wish was expressed for a body schema model for wound localization.

\section{Discussion}

The results of the evaluation reflect a positive trend that the PosiThera demonstrator can support the addressed use cases "Diagnostic process" and "Therapy support" in the care of chronic wounds. The presented evaluation results are based on a small database $(\mathrm{n}=35$ test data sets, $\mathrm{n}=14$ "freestyle scenarios) and were only performed by 5 testers. However, all test persons had practical nursing experience and 4 of them had 3 years of nursing training, so that nursing expertise in dealing with the system was available, albeit under laboratory conditions, and this was also reflected in particular in the freestyle scenarios. The chosen user-centred design was positively received by the testers in order to actively shape the CDSS. The extension of the tests with free-style scenarios was beneficial for the holistic testing of the prototype in order to approach routine use in small steps. Nevertheless, and even though the assignment of the test patient records was randomised, the results only reflect a positive trend based on the legitimations described. 


\section{Conclusion}

Just as the positive trend in the evaluation results reflects it, the reactions of the testers in the MSH Skills Lab during the on-site evaluation also reflected the positive acceptance of the prototype: "I would wish for a system like this in my nursing practice!" The chosen method of user-centre design or the participatory approach was also positively evaluated by the nursing practitioners not only in the development [8] but also in the evaluation. Even if the system requires further development and evaluation, the results prove that nursing practice also has great potential for applications of artificial intelligence in care practice.

\section{Acknowledgments:}

This study was funded by the Federal Ministry of Education and Research Germany, support program: Medizintechnische Lösungen für die digitale Gesundheits-versorgung (grant: 13GW0171C).

\section{References}

[1] Wright A, Sittig DF. A four-phase model of the evolution of clinical decision support architectures. Int J Med Inform. 2008;77(10):641-649.

[2] Liberati EG, Ruggiero F, Galuppo L, Gorli M, González-Lorenzo M, Maraldi M, Ruggieri P, Polo Friz H, Scaratti G, Kwag KH, Vespignani R, Moja L. What hinders the uptake of computerized decision support systems in hospitals? A qualitative study and framework for implementation. Implement Sci. 2017 Sep 15;12(1):113.

[3] Holbrook A, Xu S, Banting J. What factors determine the success of clinical decision support systems? AMIA Annu Symp Proc. 2003. p.862.

[4] Pavel S Roshanov, Natasha Fernandes, Jeff M Wilczynski, Brian J Hemens, John J You, Steven M Handler, Robby Nieuwlaat, Nathan M Souza, Joseph Beyene,Harriette G C Van Spall, Amit X Garg, R Brian Haynes, Features of effective computerised clinical decision support systems:meta-regressionof 162 randomised trials, BMJ 2013;346

[5] Kilsdonk E, Peute LW, Knijnenburg SL, Jaspers MW. Factors known to influence acceptance of clinical decision support systems. Stud Health Technol Inform. 2011;169:150-4.

[6] Kashfi H. Applying a user centered design methodology in a clinical context. Stud Health Technol Inform. 2010;160(2):927-31.

[7] Vredenburg K, Isensee S, Righi C. User-Centered Design: An Integrated Approach. Prentice Hall PTR, Upper Saddle River, NJ. 2002.

[8] Vogel S, Przysucha M, Wache S, Hüsers J, Hübner U, Sellemann B. Decision Support Use Cases for the Treatment of Patients with Chronic Wounds. Deutsche Gesellschaft für Medizinische Informatik, Biometrie und Epidemiologie. 63. Jahrestagung der Deutschen Gesellschaft für Medizinische Informatik, Biometrie und Epidemiologie e.V. (GMDS). Osnabrück, 02.-06.09.2018. Düsseldorf: German Medical Science GMS Publishing House; 2018. DocAbstr. 60

[9] Myers G, The Art of Software Testing, $2^{\text {nd }}$ Edition (2004), John Wiley \& Sons Ltd., ISBN-13: 9780471469124. p. 13 and 35

[10] Vogel S., Prozessbezogene, kontextsensitive Entscheidungsunterstützung und Simulation zur Therapieunterstützung am Beispiel chronischer Wunden DMEA Satellitenveranstaltung GMDS AG "Wissensbasierte Systeme in der Medizin", Conference DMEA Satellite event, 2019, doi: 10.13140/RG.2.2.17973.12006

[11] Bültemann \& Riepe, 2013, v.27, URL: http:/www.wunduhr.de/pdf/wunduhr-wie.pdf, last access: 14.01.2021 